\title{
Les choses imprévisibles et celles qui ne le sont pas
}

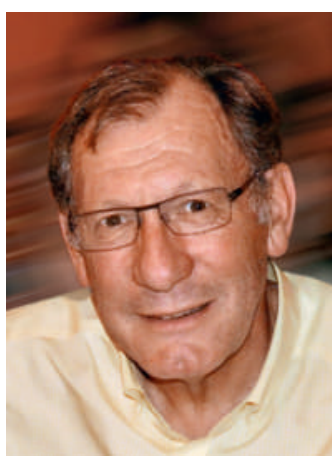

Jean Martin
Souvent on considère comme imprévisibles des évènements qui, à l'échelon d'une collectivité, sont prévisibles dans une large mesure et peuvent faire l'objet de mesures préventives. En santé publique, c'est sur la base de données scientifiques qu'on promeut de telles mesures; par exemple les gains de santé importants que permettent les vaccinations, des tests de dépistage ayant montré leur utilité, ou en rapport avec les dégâts majeurs liés au tabagisme.

Prévention aussi des accidents de la route par la ceinture de sécurité, les contrôles de l'alcoolémie, les limites de vitesse. Il importe de garder à l'esprit le fait de santé publique que «les accidents ne sont pas accidentels». Si la dimension imprévue (qui apparaît dans la définition des dictionnaires et à la table du Café du Commerce) peut être vraie pour une personne donnée, ce n'est pas vérifié dans une population. L'épidémiologie (étude statistique des circonstances des accidents et des caractéristiques des personnes concernées) identifie les facteurs de risque accru à cet égard, facteurs qui peuvent se cumuler et augmenter d'autant plus la probabilité d'un malheur.

On distingue violence involontaire (les accidents) et violence volontaire (incivilités, agressions, homicides). A propos de cette dernière: nous sommes frappés de ce côté-ci de l'Atlantique par les tueries par armes à feu qui reviennent périodiquement aux Etats-Unis, dans des écoles et campus universitaires, des supermarchés. L'impuissance des pouvoirs publics à y faire face est liée à des mythes de type Far West, du pistolet battant la hanche de tout cowboy qui se respecte... Les grands prêtres de cette imagerie «patriotique» meurtrière, au premier rang desquels la très funeste National Rifle Association (NRA), ont dans leur fonds de commerce d'arguments fallacieux l'idée que cette violence est inévitable et due principalement à la méchanceté de «bad guys» (surtout s'ils n'appartiennent pas à la majorité blanche). Sur cette base erronée d'imprévisibilité, la NRA veut que chaque résident des Etats-Unis ait à la maison un arsenal pour sa «légitime défense». Raisonnement inepte, scandaleux. Si les Etats-Unis connaissaient un système tant soit peu adéquat de contrôle des armes et de leurs détenteurs et des programmes d'éducation y relative (à la non-violence, pas au maniement des fusils!), on aurait sans délai une forte diminution de ces drames. Pourtant l'idéologie prévalente fait que le Gouvernement fédéral est incapable de faire passer des lois restrictives, même au lende- main d'un massacre d'enfants comme dans le Connecticut en décembre 2012. Cauchemar.

Quelles imprévisibilités me préoccupent-elles aujourd'hui? Les principales ne sont pas en santé publique, bien qu'elles aient des conséquences au plan sanitaire et social. Le système financier international: cinq ans après la crise qui a demandé que les pouvoirs publics volent au secours de grandes banques, beaucoup d'acteurs financiers se comportent sans tenir compte d'une quelconque responsabilité sociétale. Le monde: des guerres peuvent être déclenchées par un évènement, un casus belli, objectivement mineur. S'agissant du drame de la Syrie, détruite depuis deux ans par la guerre civile, on doit bien sûr regretter la passivité de grandes puissances. Mais la place de ce pays sur l'échiquier politico-diplomatique est telle que chacun craint qu'une intervention armée de l'extérieur corresponde à mettre le doigt dans un engrenage, avec des conséquences imprévisibles à large échelle.

Plus avant: je me suis engagé au cours des années pour la coexistence pacifique et respectueuse entre cultures, ethnies, religions et, mieux que cela, pour une convivialité, soulignant que «la différence, c'est toujours intéressant et enrichissant». En ce moment toutefois, j'avoue une inquiétude devant tant de proclamations agressives de «différences» et la montée des intolérances et extrémismes. Qui saurait répondre à la question de savoir si la déstabilisation de plusieurs régions du monde par des groupes terroristes ne va pas s'étendre de part en part? Dans tous les cas, ce que nous vivons a un caractère sérieux d'imprévisibilité. On aurait envie que les analystes experts puissent décrire/démonter les causes et mécanismes de l'instabilité actuelle, voir en quoi certains développements ne sont pas imprévisibles et conseiller les décideurs en conséquence.

Enfin, un défi d'envergure planétaire où l'évolution future et ses conséquences ne sont que trop prévisibles: le réchauffement climatique et les altérations irréversibles de l'environnement. Les rapports du GIEC sont solides et les climato-sceptiques scientifiquement pas crédibles. Là aussi, la pusillanimité des politiques à, d'abord admettre, ensuite saisir à bras le corps les problèmes laisse songeur - pour ne pas dire un peu désespéré.

Toutefois, le pire n'est jamais certain dit-on.

Jean Martin, membre de la rédaction 